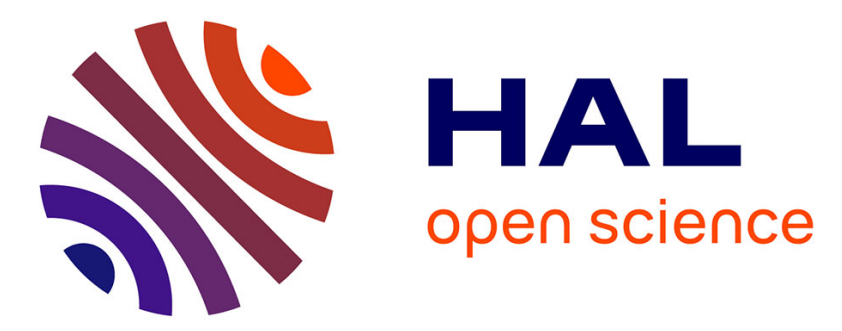

\title{
Modeling and analysis of triage nurse ordering in emergency departments
}

Karim Ghanes, Oualid Jouini, Mathias Wargon, Zied Jemai

\section{To cite this version:}

Karim Ghanes, Oualid Jouini, Mathias Wargon, Zied Jemai. Modeling and analysis of triage nurse ordering in emergency departments. Conference on Industrial Engineering and Systems Management IESM'15, Oct 2015, Seville, Spain. 10.1109/IESM.2015.7380163 . hal-01265284

\section{HAL Id: hal-01265284 \\ https://hal.science/hal-01265284}

Submitted on 31 Jan 2016

HAL is a multi-disciplinary open access archive for the deposit and dissemination of scientific research documents, whether they are published or not. The documents may come from teaching and research institutions in France or abroad, or from public or private research centers.
L'archive ouverte pluridisciplinaire HAL, est destinée au dépôt et à la diffusion de documents scientifiques de niveau recherche, publiés ou non, émanant des établissements d'enseignement et de recherche français ou étrangers, des laboratoires publics ou privés. 


\title{
Modeling and analysis of triage nurse ordering in emergency departments
}

\author{
(presented at the 6th IESM Conference, October 2015, Seville, Spain) $\mathrm{C}^{4} \mathrm{e}^{2} 2015$
}

\author{
Karim Ghanes, Oualid Jouini \\ Laboratoire Genie Industriel \\ CentraleSupélec, Université Paris-Saclay \\ Châtenay-Malabry, France
}

\author{
Mathias Wargon \\ Emergency Department \\ Saint Camille Hospital \\ Bry-sur-Marne, France
}

\author{
Zied Jemai \\ OASIS -ENIT, University of Tunis El \\ Manar \\ Tunis, Tunisia
}

\begin{abstract}
Emergency departments are facing a worldwide problem that affects their performance, namely Overcrowding. Triage Nurse Ordering appears to be a promising approach in addition to be cost effective. This paper proposes a process-based triage nurse ordering model and assesses its efficiency on the ED performance through simulation while considering the length of stay as the key indicator. The study examines the impact of triage nurse ability, system load and triage time extension on the benefits that might be derived from triage nurse ordering.
\end{abstract}

Keywords-emergency departments; triage nurse ordering; simulation; length of stay

\section{INTRODUCTION}

Emergency Department (ED) is the service within hospitals responsible for providing care to life-threatening and other emergency cases over 24 hours daily, 7 days a week. Nowadays, EDs are facing a recurrent worldwide crisis, namely overcrowding. Using various methods, healthcare practitioners and researchers in operations management try to alleviate this overcrowding.

In order to improve ED performance, an extensive body of the literature consists on introducing additional resources (physicians, beds, etc.). This includes either resource allocation issues [1,2,3] or process modification issues [4] such as some interventions in triage (team triage and physician at triage), point of care testing, etc. This is the most direct way to alleviate crowding and improve responsiveness. However, as reported in [5], because it is also the most expensive approach, it is generally not the preferred option. High salaries of doctors and high costs of medical equipments [6,7] combined to budgetary restrictions has prompted practitioners and researchers to investigate new methods that are costeffective. Among these, Triage Nurse Ordering (TNO) appears to be a promising approach that does not require any resource investment. It can be achieved using existing staff with little additional training [8].

TNO is an advanced triage intervention that consists on allowing triage nurse to order tests and treatments before the patient is seen by the physician $[9,10,11]$. The common protocol in the ED is that triage nurse cannot order diagnosis tests. He/she is essentially responsible of making a first assessment of patients' state and categorizing them into different acuity levels. The decision of requiring diagnosis tests or not is traditionally under the responsibility of the ED physician. However, the medical literature suggests that with an appropriate education and training, and adapted protocoled guidelines, triage nurses could be able to order some tests to a level comparable to that of physicians $[9,12,13,10]$. Diagnostic imaging and laboratory tests are time-consuming processes in the ED that are associated with longest length of stay (LOS) $[9,14,15]$. If tests are early requested in the triage process, they could be undergone without waiting for the first examination by the ED physician, and test results could be reviewed by the latter as soon as he becomes available.

TNO has been related to enhanced patient satisfaction $[16,17,18]$. However, "little is known about the effectiveness of this intervention in improving ED time metrics" [8]. Only few medical papers reported that TNO could possibly reduce the ED LOS $[19,20,10,18]$. As mentioned in [4], there is only limited scientific evidence that having nurses to request certain tests results in shorter waiting time and LOS. Moreover, as highlighted by [8], the existing LOS improvements revealed in the literature may range widely (from 2.45 to 74 minutes). There is a real need to conduct studies that will legitimize the use of TNO in EDS in terms of LOS reduction $[9,8]$.

In the present paper, the objective is to analyze the effect of TNO on ED time metrics taking into consideration the key parameters of such an intervention. We relied on an online survey that we performed with EDs from different countries in order to understand the current practices and obtain specialists' opinions. The survey's questions focused on the relevancy of TNO, on which types of diagnosis tests exactly it could be applied and why. This survey helped us to understand the whys and wherefores of this problem in order to delimit the framework and include the most relevant parameters related to TNO in our model. Using simulation, we assess the effectiveness of TNO as a function of triage nurse ability 
level, and investigate other elements that could have an impact on this effectiveness (System load, service time prolongation). We derive useful insights that can assist decision makers when implementing a TNO intervention.

The rest of the paper is organized as follows. A literature review on TNO is presented in section 2. In Section 3, we report the results of the performed survey and describe in detail our TNO model. In Section 4, we conduct experiments. Finally, in section 5, we summarize the main insights and highlight some future research.

\section{LITERATURE REVIEW}

TNO, also called advanced triage [21,20], is a worldwide ED issue that was addressed in North America [19,20], Europe [21,16], Asia [22,18] and Australia [17]. The prior literature examining TNO is almost exclusively addressed from a medical perspective. It consists in general on 2 types of empirical studies: examining the ability of triage nurses in initiating diagnosis tests properly [10], assessing the effect of such an intervention on ED time metrics [20,16,17], or both $[21,18]$. For the first type, the method consists generally on using the attending physician as a standard to judge the accuracy of triage nurse orders, and for the second it consists on comparing statistically two samples of patients (one with the traditional ED process and another with TNO) in terms of time metrics, mainly LOS.

As highlighted in [8] and [4], most of TNO interventions are limited to some radiographs, mostly joints and bones of distal limbs $[13,16,18]$. However, some include additional diagnostic test requesting such as blood tests, urinalysis, electrocardiogram $[20,23,10,24]$ and radiographs of other parts like the skull [22]. Even if there is some unanimity about distal limb radiographs, the choice of the diagnosis type for TNO is rarely justified in the literature and still remains unclear.

TNO has been related in some papers to a decreased LOS. Reference [21] reported that LOS decreased by 27 minutes (18\%) with foot/ankle X-rays initiated at triage. In [20], time savings was on average $46 \mathrm{~min}$ in the total LOS with TNO applied on some X-rays and blood tests. In [18], total LOS for patients with radiographs requested by the nurse was on average 18.59 minutes less than the overall average. Reference [16] reported that a mean reduction of $37.2 \mathrm{~min}$ (36\%) from time of triage to time of treatment decision was achieved in the group of patients with triage initiated X-rays comparing to control group. However, according to [8] and [9], there is a paucity of research examining the effect of TNO intervention on ED time metrics. Time reductions related to TNO may range widely (from 2.45 to 74 minutes according to [9]) and some negative conclusions have also been reported. Reference [17] performed a comparison between a group of patients who had X-ray initiated in triage and a group with a regular pathway, and found that the difference in LOS between the two groups was not statistically significant. However, staff and patient satisfaction with this change was high and justified the continuation of the practice in the ED.
Satisfaction was not formally measured but it was reported that physician's satisfaction was increased through the availability of diagnostic results since the first examination; patients seemed satisfied for using the waiting time more efficiently in addition to a greater sense of team working for all staff $[20,13,16,17]$.

One of the arguments facing TNO is the fear of overrequesting diagnostic tests that would not have been ordered by the physician [18]. The potential benefits of TNO in terms of time savings and satisfaction must be balanced with the disadvantages of such excessive requests: additional time, additional expense and increased resource utilization, unnecessary radiation exposure and potential morbidity [10,25]. For instance, [18] addressed the problem of triage nurse ability in ordering radiographs including 934 patients in their study. The triage nurse requested radiographs for $94.54 \%$ of patients (883). 5.44\% of requested tests (48 out of the 883 ) were considered unnecessary by the case physician.

Under-requesting is another type of possible error. Triage nurse could miss some necessary tests that will further be required by the physician. In [18], among the 51 cases where triage nurse did not order any radio (5.5\%), 65\% (33/51) had an X-ray requested by the attending physician (underrequesting rate of $3.5 \%, 33 / 934)$. TNO presents also a risk of additional tests following the physician's examination. Additional views of the same/adjacent or different regions can be ordered because the first view did not demonstrate the problem or another injury was discovered during the consultation $[4,26]$. "Additional trips to the radiology department become necessary, increasing both the time required for treatment and the inconvenience to the patient" [25]. In [18], 11\% of ordered radios (97 out of 883) were followed by additional ones after physician's assessment.

In addition to [18], a few other papers examined the ability of triage nurses in initiating radiographs appropriately (using similar inclusion criteria). The reported statistics from most complete studies are provided in table I. 
TABLE I. TNO ABILITY STATISTICS REPORTED IN THE LITERATURE

\begin{tabular}{|c|c|c|c|c|}
\hline & {$[18]$} & [26] & [27] & Others \\
\hline $\begin{array}{c}\text { Sample size } \\
\text { on which } \\
\text { TNO was } \\
\text { applied } \\
\end{array}$ & 934 & 579 & 915 & \\
\hline $\begin{array}{c}\text { Triage nurse } \\
\text { Requesting } \\
\text { rate }\end{array}$ & $94.5 \%$ & $\begin{array}{c}72 \% \\
(416 / 579)\end{array}$ & $78 \%$ & \\
\hline $\begin{array}{c}\text { Over- } \\
\text { requesting } \\
\text { rate }(\mathrm{N}+/ \mathrm{P}-)\end{array}$ & $\begin{array}{c}5.4 \% \text { of } \\
\text { ordered tests } \\
\text { considered } \\
\text { unnecessary } \\
\text { by physician }\end{array}$ & $\begin{array}{c}6.5 \% \text { of } \\
\text { ordered tests } \\
\text { considered } \\
\text { unnecessary } \\
\text { by physician }\end{array}$ & $\begin{array}{c}4 \% \text { of } \\
\text { ordered tests } \\
\text { considered } \\
\text { unnecessary } \\
\text { by physician }\end{array}$ & $\begin{array}{c}4.5 \% \text { and } 8 \% \\
\text { in [25] and } \\
{[21]} \\
\text { respectively }\end{array}$ \\
\hline $\begin{array}{c}\text { Under- } \\
\text { requesting } \\
\text { rate }(\mathrm{N}-/ \mathrm{P}+)\end{array}$ & $\begin{array}{l}65 \% \text { of } \\
\text { situations of } \\
\text { no tests } \\
\text { ordered by } \\
\text { nurse were } \\
\text { followed by a } \\
\text { physician } \\
\text { order ( } 3.5 \% \text { of } \\
\text { total sample) }\end{array}$ & $\begin{array}{c}47.2 \% \text { of } \\
\text { situations of } \\
\text { no tests } \\
\text { ordered by } \\
\text { nurse were } \\
\text { followed by } \\
\text { a physician } \\
\text { order } \\
(13.3 \% \\
\text { of total } \\
\text { sample) }\end{array}$ & $\begin{array}{c}23.5 \% \\
(66 / 281) \text { of } \\
\text { situations of } \\
\text { no tests } \\
\text { ordered by } \\
\text { nurse were } \\
\text { followed by } \\
\text { a physician } \\
\text { order ( } 8 \% \text { of } \\
\text { total) }\end{array}$ & \\
\hline $\begin{array}{l}\text { Rate of } \\
\text { additional } \\
\text { tests } \\
\text { requested by } \\
\text { physician } \\
(\mathrm{N}+/ \mathrm{P}++)\end{array}$ & $\begin{array}{c}11 \% \text { of already } \\
\text { x-rayed } \\
\text { patients }\end{array}$ & $\begin{array}{c}5.3 \% \\
(22 / 416) \text { of } \\
\text { already } x- \\
\text { rayed } \\
\text { patients }\end{array}$ & $\begin{array}{c}7.2 \% \text { of } \\
\text { already x- } \\
\text { rayed } \\
\text { patients }\end{array}$ & $7.8 \%$ in [16] \\
\hline
\end{tabular}

Note that when additional tests are ordered by the physician, studies did not mention whether the ones ordered by the nurse were necessary or not. No statistics were formally reported about patients with both over-requesting and underrequesting (additional tests after unnecessary tests) except in rare papers like [10] where these patients represented $15 \%$.

The success of TNO can likely be achieved using existing triage nurses with little additional training [8]. In most of the analyzed papers, triage nurses' skills were extended before experiments with training programs on examination skills and inclusion/exclusion criteria for exams requisition [20,13,18]. As demonstrated by [10], the ability of triage nurse in ordering tests can be improved with the use of test ordering guidelines. Reference [16] reports a reduced gap between triage nurse and physician ability in ordering radiographs after participating in a 1-day training program and by using carefully designed protocols. However, there are no standardized guidelines for TNO interventions [8]. Reported trainings are various in time and contents $[9,8]$. In addition to an initial non-uniformity in nurse education between countries, and also within the same country [12], different TNO trainings and protocols could have influenced results reported in studies [9,27].
As explained above, TNO contributions consisted either on measuring the impact of such an intervention on time metrics or assessing triage nurse ability. However, it should be noted that no paper analyzed or quantified the impact of nurse ability on patient time in the ED so far. Service times' prolongation is also an element that was not addressed. As mentioned in [16] and [18], the average time of triage and consultation could be lengthened under TNO which could also affect the results.

In the OR/OM domain, we identify very limited contributions. Reference [24] uses simulation to test several staffing and process alternatives in order to reduce the patients LOS in an ED (TNO, fast track, point of care testing, etc.). There was no significant time savings related to TNO (3.6 minutes on the total average LOS). However, authors suggested that TNO would be more effective when the system is quite busy, but that was not demonstrated. With the exception of the considered types of exams (X-rays, lab tests and ECG), no information was provided about the parameters included in the TNO model (changes in the patient pathway, triage nurse ability, etc.).

\section{MODEL DEFINITION}

In order to understand specialists' opinions about TNO and define the appropriate model framework, a survey was performed in many EDs in France, USA, Netherlands, Germany, Belgium, Greece and Tunisia.

\section{A. Survey results}

The results are based on 36 practitioners (ED managers and ED physicians) from 28 different EDs. 75\% of the answers were provided by specialists from French EDs. The majority of surveyed specialists $(86 \%)$ considered TNO as a potential relevant practice in general. However as shown in table II, the feasibility of TNO varies greatly from one test type to another. For each one, the experts provided the practical reasons about the possibility to apply TNO or not.

TABLE II. THE POSSIBILITY TO APPLY TNO FOR THE MAIN TYPES OF TESTS

\begin{tabular}{|c|c|}
\cline { 2 - 2 } \multicolumn{1}{c|}{} & $\begin{array}{c}\text { For which types of tests could TNO } \\
\text { be relevant? }\end{array}$ \\
\hline X-ray & $\mathbf{9 7 \%}$ \\
\hline CT-scan & $3 \%$ \\
\hline MRI & $0 \%$ \\
\hline Echo & $0 \%$ \\
\hline Blood tests & $47 \%$ \\
\hline Urine analysis & $83 \%$ \\
\hline ECG & $83 \%$ \\
\hline
\end{tabular}

Conventional radiology, also called standard radiology, radiographs or X-rays, were considered by the experts as the most appropriate diagnosis tests for a TNO intervention in their EDs. Only some particular types of radiographs are 
concerned which are those for simple extremity traumatology of stable patients. That includes bones and joints radiographs of distal limbs that are below the large joints like the hip and the shoulder (hand, wrist, elbow, foot, knee, ankle, etc.). They are routine tests, easy to perform with limited risk for patients (noninvasive). Multi trauma cases as well as radiographs of other parts such as spine, chest, abdomen and pelvis should be excluded.

For many reasons, CT-scan, MRI and Echo were judged inappropriate for a TNO intervention. They are much more expensive and represent critical resources in the hospital. The application of TNO on these tests requires a medico-economic evaluation. Moreover, they are invasive and more specific tests. The decision of ordering such tests is complex and cannot be done without a complete (physical and clinical) examination by a physician.

Biological tests are also complex and costly and the opinion of experts about the application of TNO on them is mixed. The survey revealed $83 \%$ of favorable views for TNO of urinalysis. However, that was limited by specialists to certain basic urine tests. The mainly mentioned candidate is a type of urine analysis called urine test strip. It is a basic and quick diagnosis tool that is used by practitioners in the ED without even resorting to the laboratory. Other kinds of basic urine tests were mentioned such as urine pregnancy tests. According to the survey, blood tests can be ordered by triage nurse only in certain cases (fever in a patient back from a tropical country or in a patient receiving chemo, diabetes, HIV testing, etc.). More specific and sophisticated blood tests require a clinical examination by a physician and must be discussed on a caseby-case basis. The disparity in the situations requiring biological testing makes them difficult to generalize.

There is unanimity on allowing triage nurse to decide about an Electrocardiogram (ECG) in several cases like chest pain. This protocol is more common than other tests and is already applied in many surveyed EDs.

\section{B. The model description}

Given the answers collected from the performed survey, the study will focus on low acuity level patients (ESI4 and ESI5) with distal limbs traumatology requiring conventional radiographs (X-rays). In France, Trauma injuries represent about half of ED visits $[28,29]$. Among these, trauma to the extremity whether upper like wrist and hand injuries, or lower like ankle sprain represent the most common cases in EDs particularly among non-critical patients, with X-rays being the reference test [30].

For this kind of patients, length of stay (LOS) is the key metric $[31,5]$. "Due to relatively minor nature of these injuries, those patients have often to wait a long time for treatment and investigation in EDs" [17]. This group of patients rarely requires biological tests which make them free of any necessary sampling, and allow sending them to radiology right after triage.

The interviews with experts combined with the existing literature allowed to identify the most relevant parameters that could have an impact on the effectiveness of TNO, and that will further constitute the basis of our experiments:

- The accuracy of triage nurse in requesting tests (over, under and incomplete-requesting)

- The ED level of crowding

- The impact of TNO on some service times

As shown in Fig.1, comparing to the traditional patient pathway, when triage nurse orders diagnosis tests, they are initiated right after triage. Since ordering tests is an additional task for triage nurse, the triage service time represented in our model by a random variable $\mathrm{T}$ could be increased by a certain amount of time $\Delta \mathrm{T}$. When tests are completed, the physician examines the patient for the first time and interprets his/her tests results during one single aggregated consultation. In our model, we make the assumption that this task has the same time distribution with a regular consultation.

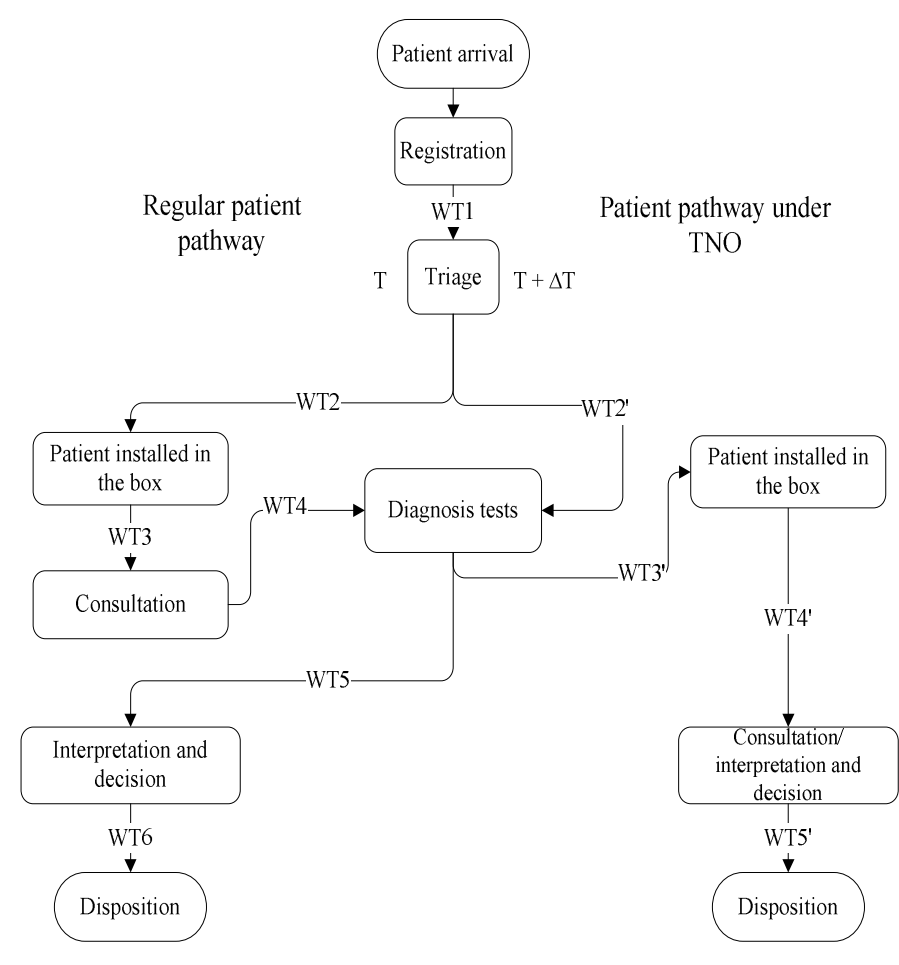

FIGURE 1: REGULAR AND TNO PATIENT PATHWAY

The TNO path depicted in Fig.1 is a simplified representation that corresponds to a particular ideal scenario under TNO (see N+/P+ in Fig.2). According to the case and the triage nurse risk of error, we distinguish in total between six possible situations. The latters are depicted with the appropriate formalism in Fig. 2. 


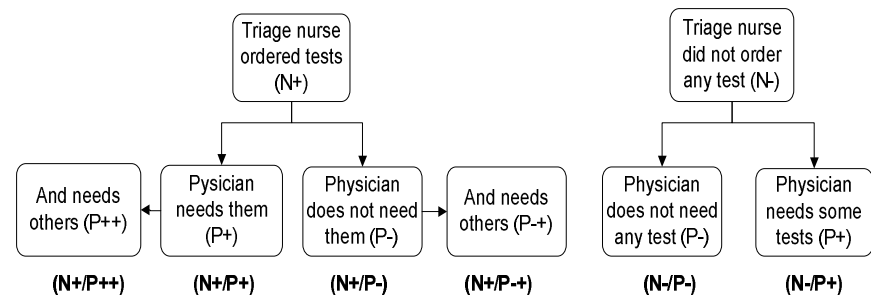

FIGURE 2: POSSIBLE SITUATIONS UNDER TNO

$(\mathrm{N}+/ \mathrm{P}+)$ is the ideal scenario that embodies the utility of TNO. Triage nurse orders the appropriate tests which would allow saving the time of a first consultation and its corresponding waiting times (WTs). In scenario (N-/P-), the nurse is right and this situation has neither advantages nor disadvantages. $(\mathrm{N}-/ \mathrm{P}+)$ is a harmless situation of underrequesting where the TNO patient pathway is unintentionally turned into a regular one.

The rest of the scenarios are considered negative and would generate loss of time because of nurse errors made in ordering tests. ( $\mathrm{N}+\mathrm{P}-)$ is a situation of over-requesting where time is lost for unnecessary tests; while without TNO, the patient would have been discharged right after the first consultation. $(\mathrm{N}+/ \mathrm{P}++)$ is a particular situation of under-requesting where triage nurse orders some necessary tests while missing others (the physician may order radiographs of other parts or different views of the same), which imposes additional trips to radiology. $(\mathrm{N}+/ \mathrm{P}-+)$ is a combination of both under and over requesting in which the patient is first sent unnecessarily to radiology by triage nurse, and then sent back later by the physician. In addition to potential loss of time, these situations have an impact on patient's convenience and satisfaction.

As mentioned in the literature review, in empirical studies, reported statistics on the two situations $(\mathrm{N}+/ \mathrm{P}++)$ and $(\mathrm{N}+/ \mathrm{P}-$ $+)$ are merged. Thus, they will also not be differentiated in our model and will all be considered as $(\mathrm{N}+/ \mathrm{P}++)$. In other words, we make the assumption that when the physician orders additional tests, the ones ordered by triage nurse are never completely useless. Consequently, we obtain five possible patient paths under TNO that are represented in Fig.3.

\section{EXPERIMENTS}

Our experiments consist on a case study involving a French ED (Saint-Camille) and are divided into 4 parts. In the first one, the objective is to understand the impact of nurse's abilities and decisions on TNO effectiveness. We calculate the expected improvement in LOS as a function of a realistic range of TNO-related probabilities. In the second part, we extend the analysis by varying the different probabilities within a wider range of values in order to figure out what is the most harmful nurse error. In the third part, we assess the relationship between the ED load and TNO effectiveness. Finally, we assess the impact of triage processing time prolongation on the system performance.
Collected data from Saint Camille indicate that eligible patients represent $17 \%$ of the total number of patients. A realistic discrete event simulation model is used to perform our experiments on Saint Camille ED [32]. In this model, all common structural and functional characteristics of EDs, at least in France, were taken into consideration thanks to a close collaboration with practitioners.

For each simulation, we use one replication of 100 weeks with a warm-up period of one week.

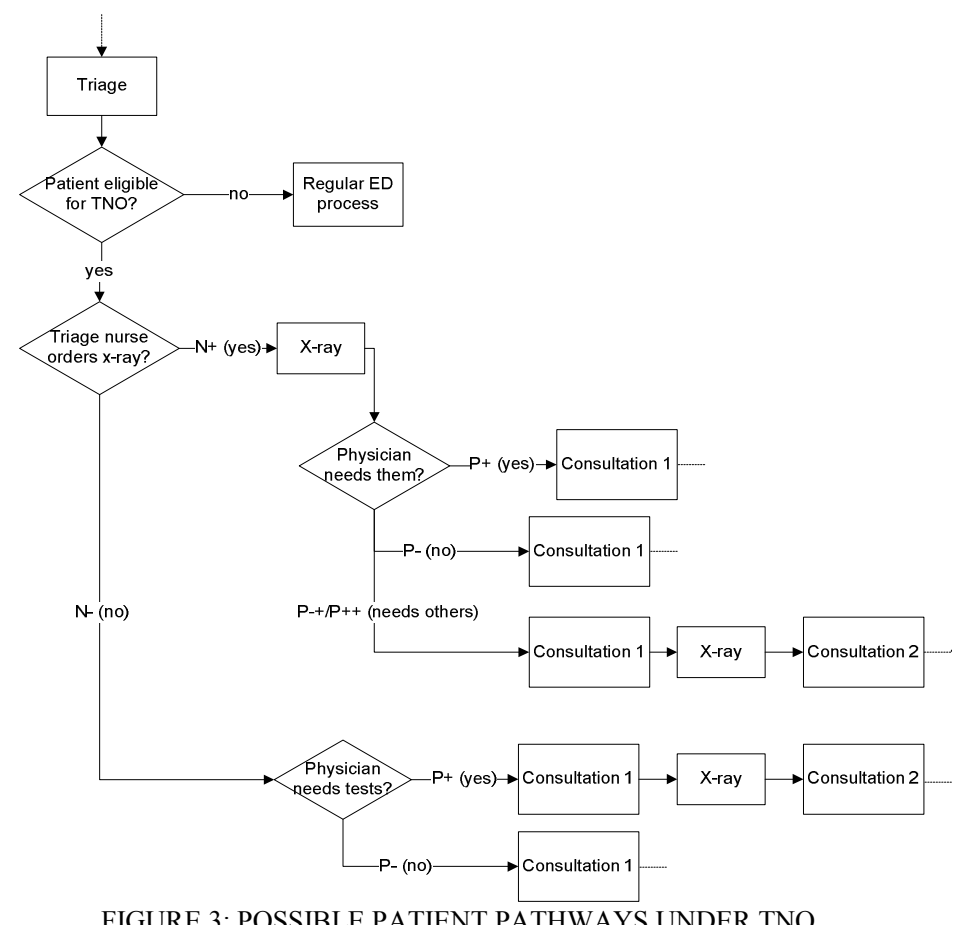

FIGURE 3: POSSIBLE PATIENT PATHWAYS UNDER TNO

\section{A. The impact of realistic trained triage nurse's ability on LOS}

We identified 4 key probabilities related to a TNO intervention. The first one is Triage nurse requesting rate. It represents the rate of patients sent to radiology by triage nurse among all eligible patients for TNO. The three other probabilities characterize triage nurse ability and her precision in requesting tests: Over-requesting rate $(\mathrm{N}+/ \mathrm{P}-)$, Underrequesting rate $(\mathrm{N}-/ \mathrm{P}+)$ and the Rate of additional tests requested by physician $(\mathrm{N}+/ \mathrm{P}++)$. For each rate, we use the lowest and the highest value found in the literature (see table 1) and generate all the possible combinations $\left(2^{4}=16\right.$ in total). For instance, the scenario H-LLH refers to a TNO intervention where triage nurse has a high requesting rate, a low probability of over-requesting, a low probability of under-requesting and a high probability to require incomplete tests (that will be followed by a physician test order). The LOS improvement of each scenario for eligible patients is depicted in Fig.4 and Fig.5. 


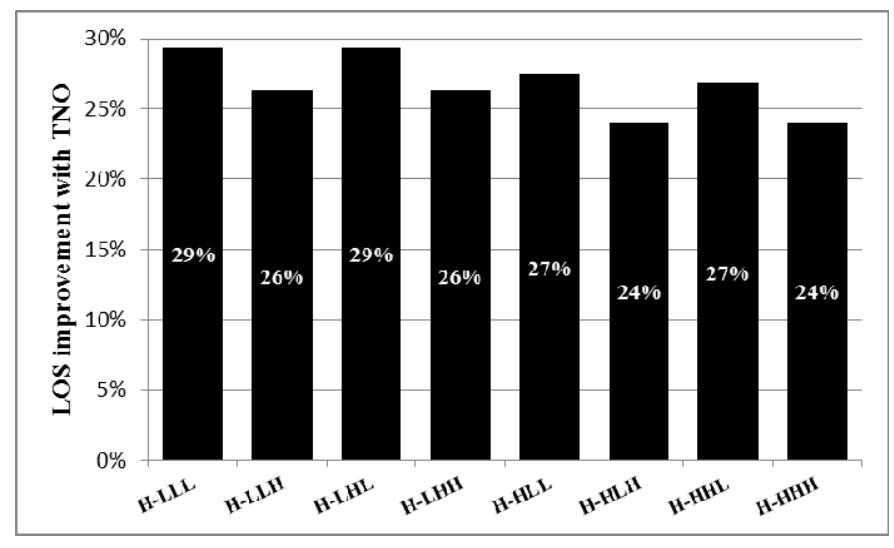

FIGURE 4: LOS IMPROVEMENT WITH TNO FOR HIGH REQUESTING RATES SCENARIOS

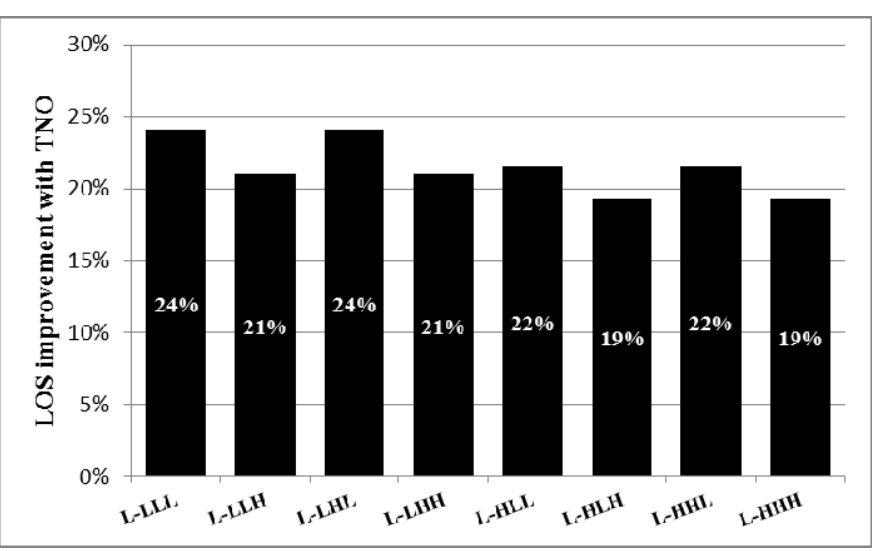

FIGURE 5: LOS IMPROVEMENT WITH TNO FOR LOW REQUESTING RATES SCENARIOS

The derived insights can be summarized as follows:

- Within the used ranges of trained triage nurses' ability reported by empirical studies, TNO is a beneficial intervention for all combinations.

- For any given trained nurse ability (for any set of over/under/incomplete requesting rate), trained triage nurse should preferably order tests as much as possible while respecting the predetermined protocols.

- Under-requesting appears to be harmless. Over and incomplete requesting both reduces the benefit from TNO.

The worst scenarios are the situations L-HHH and L-HLH (19.31\% among eligible patients and $4.49 \%$ of overall improvement) where triage nurse has the worst abilities while having a low requesting rate. The best scenario is the opposite situations H-LLL and H-LHL (29.38\% among eligible patients and $7.65 \%$ overall).

\section{B. Extended analysis of TNO effectiveness as a function of the key probabilities}

So far, no conclusion can be drawn about which triage nurse's ordering error is the most harmful. This is because the used error probabilities coming from the literature are limited. They correspond to the ability of triage nurses that were preliminarily trained. In what follows we will experiment the best scenario H-LLL by varying one by one each probability rate from $0 \%$ to $100 \%$.

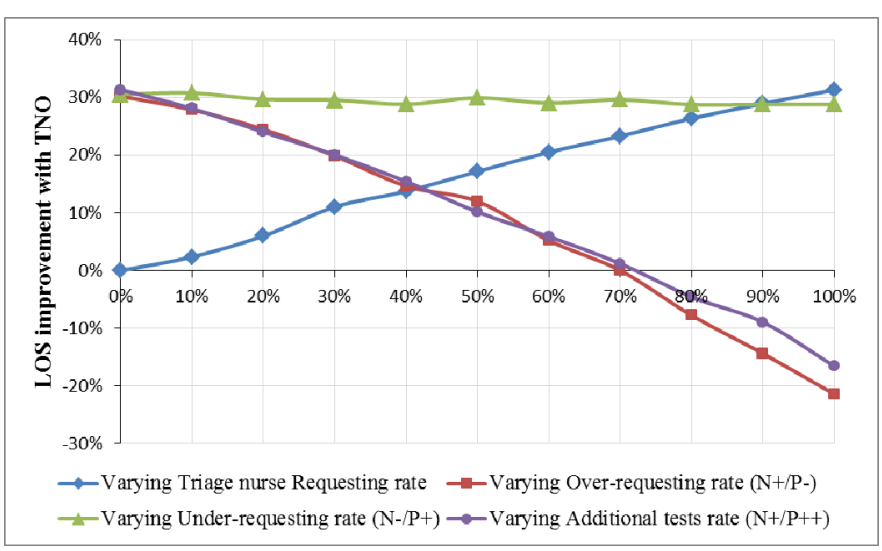

FIGURE 6: SENSITIVITY ANALYSIS ON THE 4 KEY PROBABILITIES

The following insights can be derived:

- The benefit from TNO is more apparent for higher requesting rates (with respect to protocols).

- The risk of under-requesting rate has no impact on TNO performance

- The risk of over-requesting and the risk of incomplete requesting (additional tests further requested by physician) affect TNO performance and have similar impacts on it. This result is quite intuitive since both of them consist of an additional trip to radiology department. This result holds under the assumption that when the physician orders additional tests, the ones ordered by triage nurse were not completely useless. Otherwise, the rate of additional tests would be the most harmful.

- For over and incomplete requesting rates, there is a threshold under which TNO could be detrimental for the system performance.

\section{TNO effectiveness as a function of the system load}

The actual arrival pattern in Saint-Camille ED depends on the day of the week and the hour of the day. Similarly to [33] and [34], we assume that arrivals follow a non-homogenous Poisson process ( $7 \times 24$ arrival rates).

In order to assess the relationship between the system workload and the expected benefit from TNO, we perform a sensitivity analysis by varying arrival rates, in the best and the worst scenarios of Section A (see Fig.7). 


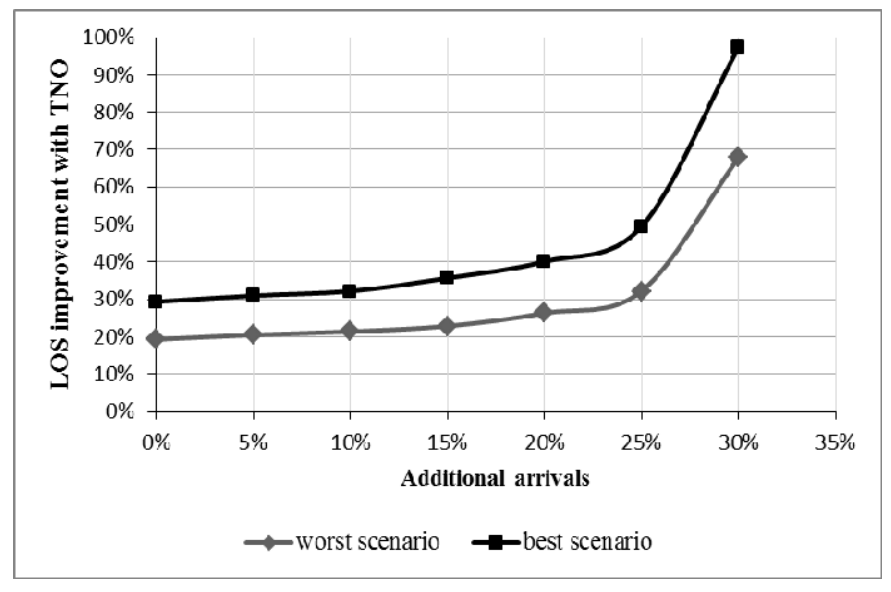

FIGURE 7: TNO EFFECTIVENESS DEPENDING ON ARRIVALS

The following conclusion can be drawn: The benefit derived from TNO is more apparent for heavily loaded EDs.

\section{The impact of triage service time extension on TNO effectiveness}

In what follows we address the question of triage service time prolongation because of TNO and assess its impact on TNO effectiveness. According to data collection (experts' judgment in particular), the distribution of triage service time is assumed to be Normal $(7,1.5)$. Using the best and the worst scenarios from part 1 (H-LLL and L-HHH respectively), we perform a sensitivity analysis on triage time by extending it up to $200 \%$ (see Fig. 8 ).

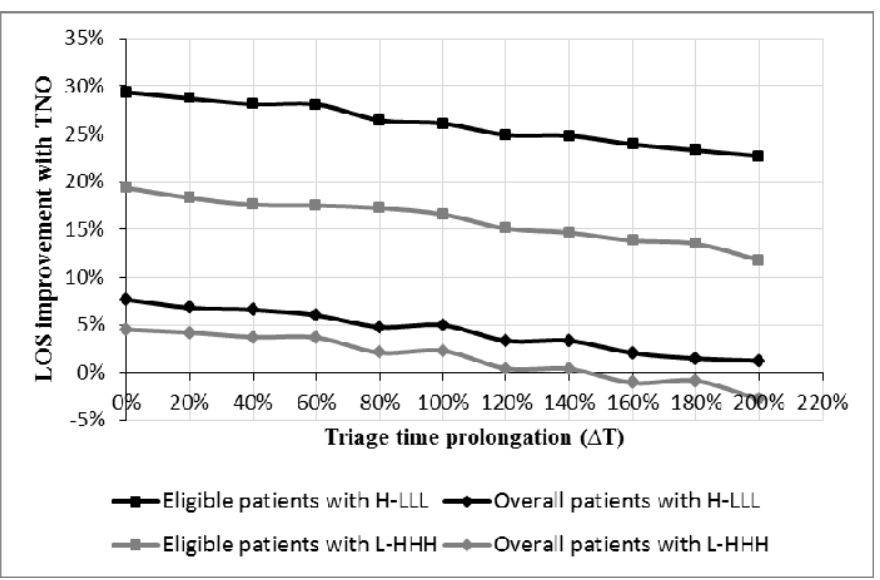

FIGURE 8: TNO EFFECTIVENESS DEPENDING ON ARRIVALS

We derive the following insights:

- $\quad$ Triage time prolongation lowers TNO effectiveness, but should not be considered as a major concern for reasonable time prolongations and nurse abilities.

- Triage time prolongation can make TNO detrimental for the overall system. For instance, for limited triage nurse abilities (L-HHH) and high triage prolongations (from $150 \%$ ), TNO remains beneficial for eligible patients but the overall system LOS is affected by longer waiting and processing times for triage.

\section{CONCLUSION}

The present paper represents the interface and the link between the two commonly addressed TNO issues, namely the assessment of triage nurse ability and the assessment of the effectiveness of this intervention in improving ED time metrics. We derived useful insights that will help decision makers for a successful implementation of TNO. For instance, TNO is always beneficial within a reasonable range of triage nurse ability level. However, there is a threshold on this ability under which TNO could be detrimental for the system performance. The benefit derived from TNO is more apparent for heavily loaded EDs. We also demonstrate that triage time prolongation does not have a significant impact on eligible patients but can affect the rest of the ED patients and the overall ED performance. Although the modeling is based on a specific ED, qualitative conclusions hold for other ED frameworks. As a perspective, an analytical modeling for TNO would be helpful for the generalization of the aforementioned results.

\section{REFERENCES}

[1] C. Duguay, and F. Chetouane, "Modeling and Improving Emergency Department Systems Using Discrete Event Simulation," Simulation. 2007; vol. 83 (4): pp.311-20.

[2] B. Bucheli, and B. Martina, "Reduced length of stay in medical emergency department patients: a prospective controlled study on emergency physician staffing," European Journal of Emergency Medicine. 2004; vol.11(1), pp.29-34.

[3] M. Rossetti, G. Trzcinski and S. Syverud, "Emergency department simulation and determination of physician staffing schedules," Proceedings of the 1999 Winter Simulation Conference.

[4] S. Oredsson, H. Jonsson, J. Rognes, L. Lind, K. Göransson, A. Ehrenberg, K. Asplund, and M.Castrén, "A systematic review of triage-related interventions to improve patient flow in emergency departments," Scandinavian Journal of Trauma, Resuscitation and Emergency Medicine. 2011; 19(43).

[5] S. Saghafian, J.W. Hopp, M.P. Van Oyen, J.S. Desmond, and S.L. Kronick, "Patient Streaming as a Mechanism for Improving Responsiveness in Emergency Departments," Operations Research. 2012; vol. 60 (5): pp.1080-97.

[6] D. Sinreich, and Y. Marmor, "Emergency Department Operations: The Basis for Developing a Simulation Tool," IIE Transactions. 2005; vol. 37 (3): pp.233-45.

[7] M. Warner, "Personnel staffing and scheduling, in Patient Flow: Reducing Delay in Healthcare Delivery," Hall, R.W. (Ed.), Springer.

[8] B.H. Rowe, C. Villa-Roel, X. Guo, M. J. Bullard, M. Ospina, B. Vandermeer et al., "The role of triage nurse ordering on mitigating overcrowding in emergency departments: a systematic review," Academic Emergency Medicine. 2011; vol. 18(12), pp.1349-1357.

[9] D.J. Robinson, "An integrative review: Triage protocols and the effect on ED length of stay," Journal of Emergency Nursing. 2013; vol. 39 (4), pp.398-408.

[10] D.C. Seaberg, B.A. MacLeod, "Correlation between triage nurse and physician ordering of ED tests," Am J Emerg Med. 1998; vol.16: pp.8-11. 
[11] A. Pallin and R.P. Kittell. "Mercy Hospital: simulation techniques for ER processes,” Industrial Engineering. 1992; vol. 24(2): pp.3537.

[12] B. Free, G.A. Lee and A. Bystrzycki. "Literature review of studies on the effectiveness of nurses ability to order and interpret Xrays," Australasian Emergency Nursing Journal. 2009; vol. 12(1): pp.8-15.

[13] M. Fry, "Triage nurses order x-rays for patients with isolated distal limb injuries: a 12-month ED study," J Emerg Nurs. 2001; vol. 27: pp. 17-22.

[14] I. Vegting, P.W. Nanayakkara, A.E. van Dongen, E. Vandew alle, J. van Galen, J. Kramer et al., "Analysing completion times in an academic emergency department: coordination of care is the weakest link," The Netherlands journal of medicine. 2011; vol. 69(9): pp.392-398.

[15] P. Yoon, I. Steiner and G. Reinhardt, "Analysis of factors influencing length of stay in the emergency department," CJEM. 2003; vol. 5(3): pp.155-61.

[16] M. Lindley-Jones and B.J. Finlayson, "Triage nurse requested xrays: are they worthwhile?" J Accid Emerg Med. 2000; vol. 17(2): pp.103-7.

[17] W. Parris, S. McCarthy, A.M. Kelly and S.Richardson, "Do triage nurse initiated X-rays for limb injuries reduce patient transit time?" Accid Emerg Nurs. 1997; vol. 5(1): pp.14-5.

[18] K.M. Lee, T.W. Wong, R. Chan, C.C. Lau, Y.K. Fu, K.H. Fund, "Accuracy and efficiency of X-ray requests initiated by triage nurses in an accident emergency department," Accid Emerg Nurs. 1996; vol. 4(4): pp.179-81.

[19] R. Retezar, E. Bessman, R. Ding, S.L. Zegar and M. McCarthy, "The effect of triage diagnostic standing orders on emergency department treatment time," Ann Emerg Med. 2011; vol. 57(2): pp.89-99.

[20] W.W.H. Cheung, L. Heeney, J.L. Pound, "An advance triage system,” Accid Emerg Nurs. 2002; vol. 10(1): pp.10-6.

[21] R.W. Rosmulder, J.J. Krabbendam, A.H. Kerkhoff, E.R. Schinkel, L.F. Beenen, and J.S. Luitse, "Advanced triage improves patient flow in the emergency department without affecting the quality of care," Nederlands tijdschrift voor geneeskunde. 2009;154, A1109.

[22] T.K. Ching, L.Y. Leng and N.B. See, "Initiation of X-rays by the triage nurse: competency and its effect on patients' total time spent in the accident and emergency department," Singapore Nurs J. 1999; vol. 26: pp.22-6.

[23] K.D. Winn, "Emergency Department Efficiency Through Utilization of Triage Nurse Protocols," Dissertation / thesis. 2001. Available at: http://en.scientificcommons.org/2274833.

[24] Kirtland, J. Lockwood, K. Poisker, L. Stamp, P. Wolfe, "Simulating an emergency department 'is asmuch fun as. . .' Proceedings of the 1995 Conference on Winter Simulation. pp. 1039-1042.

[25] C.L. McArthur and M. Thomas, "Comparison of triage nurse versus emergency physician ordering of extremity radiographs," American Journal of Emergency Medicine. 1995, vol.13 (2): pp. $248-250$

[26] A.J. Macleod and P. Freeland, "Should nurses be allowed to request X-rays in an accident \& emergency department?" Archives of Emergency Medicine. 1992, vol.9, pp.19-22.

[27] J. Thurston and S. Field, "Should accident and emergency nurses request radiographs? Results of a multicentre evaluation," J Accid Emerg Med. 1996, vol.13, pp.86-89.

[28] G. Potel, D. Lauque, J. Bouget, J.L. Lejonc, L. DeDecker, P.H. Bourrier, G. Bleichner, A. Bellou, J.P. Fournier et al, «L'organisation de l'aval des urgences: état des lieux et propositions,» SFMU. 2005.

[29] V. Carrasco and D. Baubeau, «Les usagers des urgences : premiers résultats d'une enquête nationale. Etudes et résultats 2003,» 212.

[30] O. Ganansia, «Quand ne pas prescrire de radio en traumatologie des extrémités chez l'adulte,» SFMU. 2014.

[31] K. Ghanes, A. Diakogiannis, O. Jouini, Z. Jemai, and M. Wargon. "Key performance indicators for emergency departments: A survey from an operations management perspective," Working Paper, Ecole Centrale Paris. 2014

[32] K. Ghanes, M. Wargon, O. Jouini, Z. Jemai, A. Diakogiannis, R Hellmann, V. Thomas and G. Koole, "Simulation-based optimization of staffing levels in an emergency department," Working Paper, Ecole Centrale Paris. 2014.

[33] G. Yom-Tov, and A. Mandelbaum, "Erlang-r: A time-varying queue with reentrant customers, in support of healthcare staffing," Manufacturing \& Service Operations Management. 2014; vol. 16(2): pp. 283-299

[34] M. Ahmed, and T. M. Alkhamis, "Simulation optimization for an emergency department healthcare unit in Kuwait," European Journal of Operational Research. 2009, vol. 198(3): pp. 936-942. 